\title{
The influence of elastic deformations in high-strength structural materials on the hydrogen transport
}

\author{
Polina Grigoreva ${ }^{1,2, *}$, Elena Vilchevskaya ${ }^{1,2}$, Vladimir Polyanskiy $^{1}$ \\ ${ }^{1}$ Institute for Problems in Mechanical Engineering RAS, V.O., Bolshoj pr., 61, St.-Petersburg, 199178 Russia \\ ${ }^{2}$ Peter the Great Saint-Petersburg Polytechnic University, Polytekhnicheskaya, 29, St.-Petersburg, 195259 Russia
}

\begin{abstract}
In this work, the diffusion equation for the gas-solid system is revised to describe the nonuniform distribution of hydrogen in steels. The first attempt to build a theoretical and general model and to describe the diffusion process as driven by a chemical potential gradient is made. A linear elastic solid body and ideal gas, diffusing into it, are considered. At this stage, we neglect any traps and non-linear effects. The coupled diffusion-elastic boundary problem is solved for the case of the cylinder under the tensile loads. The obtained results correspond to the experimental ones. Based on them, the assumptions about the correctness of the model and its further improvement are suggested.
\end{abstract}

\section{Introduction}

The problem of hydrogen embrittlement is a well-known one [1]: the adsorption and transport of the hydrogen from the external environment lead to the degradation of the mechanical properties of the steel. To describe the process of hydrogen diffusion from the external environment into the steel, one cannot be satisfied with only Fick's laws of diffusion, as many side effects begin to play a significant role ([2-4]). Various approaches, taking such effects into account, exist. These are models of diffusion with trapping ([5-7]), various models of stress-assist diffusion $([8,9])$, models with development of local plasticity $([10,11])$ and models of multichannel diffusion ([5-6, 12]). The already existing models describe particular experiments, usually are phenomenological and do not take into account all the factors affecting the hydrogen absorption. Moreover, these models are verified on small concentration gradients and cannot be used to describe recent experimental results.

In this work, the linear non-equilibrium thermodynamic approach is used. Considering the gradients of chemical potentials as thermodynamic forces, the diffusion equation for the system of gas and solid is written. As we aim to build a general and theoretical model, we start with the simplest case and neglect plasticity, multichannel diffusion and trapping, which in case of non-equilibrium thermodynamics approach can be simply added as terms in chemical potential. For now, the linear-elastic body is considered, without any plastic stresses or strains (which corresponds to the situation when stresses in steel, induced by the hydrogen diffusion, do not exceed flow stress). The absence of trapping and multichannel diffusion corresponds to low temperatures of diffusion.
The boundary problem for the case of the cylinder under tensile stress and diffusing hydrogen is solved to investigate if one can obtain an experimentally observed "boundary layer" - a large hydrogen concentration gradient near the outer boundary of the body ([13]) in this simplest case of such a diffusion.

\section{Diffusion problem}

\subsection{Chemical potential theory}

Following the non-equilibrium linear thermodynamics, we define the diffusion flux as

$$
\boldsymbol{j}=\frac{D c}{R T} \nabla \mu
$$

where $D$ is a diffusion constant, $R$ is a gas constant, $T$ is temperature, $c$ is a concentration and $\mu$ is chemical potential. The chemical potential for the ideal gas is equal to

$$
\mu_{g}=\mu_{0}(T)+R T \ln \frac{c}{c_{*}}
$$

where $c_{*}$ - some reference concentration. As the diffusion of hydrogen is affected by the stress-strain state of the solid body, the chemical potential of solid will also appear as a term in chemical potential of the system. In the case of the linear elastic body, the chemical potential of the solid is equal to:

$$
\mu_{s}=\frac{M}{\rho}\left(\eta(T)+\frac{1}{2}\left(\boldsymbol{\varepsilon}-\boldsymbol{\varepsilon}_{*}\right) \cdot \boldsymbol{C} \cdot \cdot\left(\boldsymbol{\varepsilon}-\boldsymbol{\varepsilon}_{*}\right)\right)
$$

where $M$ is a molar mass of the solid, $\rho$ is its density, $\eta(T)$ is chemical energy, $\boldsymbol{\varepsilon}$ is a strain tensor, $\boldsymbol{C}$ is a stiffness tensor and $\boldsymbol{\varepsilon}_{*}$ is a tensor of deformations,

* Corresponding author: polina.grigoreva239@gmail.com 
caused by the diffusion of gas into the solid body. For simplicity, it is considered that these strains depend on gas concentration linearly and they are isotropic:

$$
\boldsymbol{\varepsilon}_{*}=\alpha c \boldsymbol{E}
$$

$\alpha$ is a constant, determined experimentally.

Thus, finally, the diffusion flux is equal to

$$
\boldsymbol{j}=\frac{D c}{R T} \nabla\left(\mu_{g}+\mu_{s}\right)
$$

The diffusion equation is equal to

$$
\frac{\partial c}{\partial t}=\nabla \cdot \boldsymbol{j}
$$

After substituting and opening brackets the diffusion equation is finally obtained:

$$
\frac{\partial c}{\partial t}=\nabla \cdot\left(D_{e f f} \nabla c+v c\right)
$$

where

$$
\begin{gathered}
D_{e f f}=D\left(1-\frac{3 k \alpha c M}{R T \rho}(\operatorname{tr} \varepsilon-3 \alpha c)\right) \\
v=\frac{D M}{R T \rho}(\lambda \operatorname{tr} \varepsilon \nabla(\operatorname{tr} \varepsilon)-3 k \alpha c \nabla(\operatorname{tr} \varepsilon)+\mu \nabla(\varepsilon \cdot \cdot \varepsilon))
\end{gathered}
$$

Here $\lambda$ - Lamé's first parameter, $\mu$ is shear modulus and $k=\lambda+\frac{2}{3} \mu$ is the bulk modulus of solid.

\subsection{Boundary problem}

A cylindrical body $z \in[-h, h] ; r \in\left[0, r_{0}\right]$ is considered (see Fig. 1).



Fig. 1. The cylindrical sample under axial loads.

We prescribe axial loads $\left(\left.\sigma_{z}\right|_{z= \pm h}=\sigma_{0}\right)$. We say that the side surface is free of any load $\left(\left.\sigma_{r}\right|_{r=r_{0}}=0\right)$. The diffusion flux propagates only in $r$-direction, thus, $c=$ $c(r)$. As a boundary for the concentration, we prescribe diffusion flux at the outer surface of the body, $\left.\frac{\partial c}{\partial r}\right|_{r=r_{0}}=$ $q_{0}$. As absorption of hydrogen causes inner strains and stresses in the bulk affect the propagation of gas, the coupled problem of mutual influence of hydrogen concentration and stress-strain state of the steel is solved. To define linear stresses and strains, the Hooke's law is used:

$$
\boldsymbol{\sigma}=\lambda \operatorname{tr}\left(\boldsymbol{\varepsilon}-\boldsymbol{\varepsilon}_{*}\right) \boldsymbol{E}+2 \mu\left(\boldsymbol{\varepsilon}-\boldsymbol{\varepsilon}_{*}\right)
$$

We do not have any dynamics in our system, thus, the elasticity problem is quasistatic:

$$
\nabla \cdot \sigma=0
$$

This problem is solved numerically.

\section{Results}

To solve the coupled boundary problem we used parameters, corresponding to the real hydrogen and steel. There was no need to use exact parameters because our main goal is to see how the model takes into account stress-strain state and if it is appropriate to describe high hydrogen concentration gradients and the so-called boundary layer.

At first, the dependence of concentration on time at various points through the bulk is presented (Fig. 2). The bigger the ratio $r / r_{0}$ is, the closer the point is to the $r_{0}$. One can see that with increasing time, the concentration propagation retards even far from the outer boundary. It is also noticeable that moving away from the outer boundary, the gas propagation slows down non-linearly.

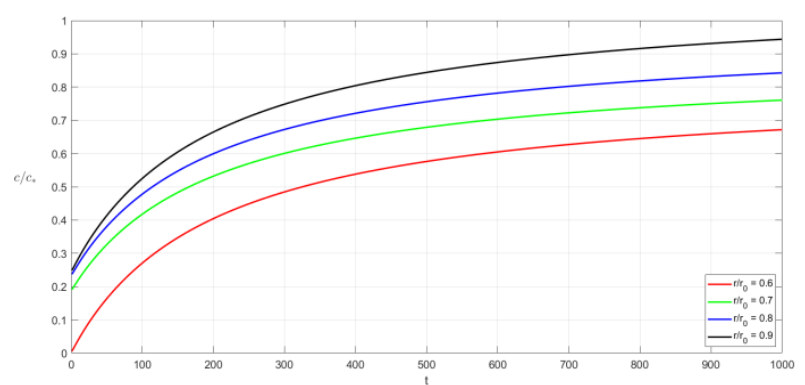

Fig. 2. The dependence of concentration on time at various $r / r_{0}$.

The dependence of concentration on radius at various times (Fig. 3) is also presented. One can see that the concentration growth slows down with time. While moving from the boundary to the centre of the sample, the concentration decreases non-linearly.

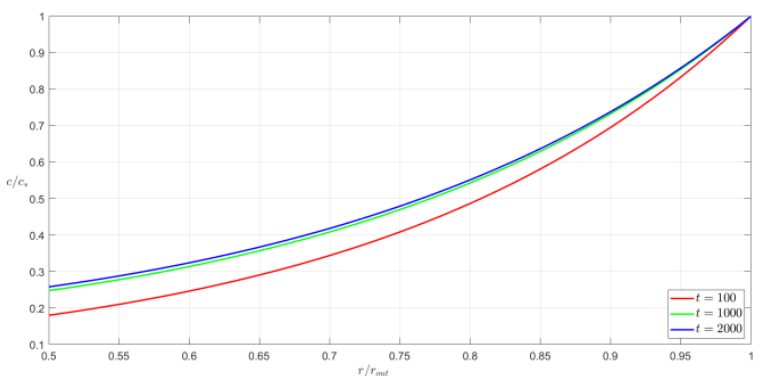

Fig. 3. The dependence of concentration on radius at various $t$.

\section{Conclusions}

Within the framework of linear nonequilibrium thermodynamics, a modified local equation for the balance of the diffusion component was obtained. This diffusion equation takes into account the mutual 
influence of diffusion and the stress-strain state, as well as the dependence of the diffusion process on temperature, gas concentration and other thermomechanical loads. The derivation of the diffusion equation allows considering all these thermomechanical effects in general. The coupled boundary value problem of determining the stress-strain state of a cylindrical specimen under axial tension and distribution of gas concentration was solved numerically. It was shown that, within the framework of the proposed model, the diffusion process passes rather quickly to a stationary regime, and the profile of the distribution of the hydrogen concentration has a pronounced nonlinear character, demonstrating a significant decrease in the hydrogen concentration with distance from the boundary to the centre of the sample. The uneven distribution of hydrogen concentration over the sample is a consequence of internal stresses arising in the material during diffusion. The results show that the model can be used to describe high gradient hydrogen concentrations. The next step could be modelling of traps, multichannel diffusion or taking into account the effects of plasticity.

The reported study was funded by RFBR, projects number 20-08-01100 A, 18-08-00201 A, project 19-38-90298.

\section{References}

1. Cailletet, L. "First report of $\mathrm{H}$ embrittlement of metals." Compt. Rend 58 (1864): 327

2. Hadam, U., and T. Zakroczymski. "Absorption of hydrogen in tensile strained iron and high-carbon steel studied by electrochemical permeation and desorption techniques." International Journal of Hydrogen Energy 34.5 (2009): 2449-2459

3. Turnbull, A. "Perspectives on hydrogen uptake, diffusion and trapping." International Journal of Hydrogen Energy 40.47 (2015): 16961-16970

4. Oudriss, A., et al. "Grain size and grain-boundary effects on diffusion and trapping of hydrogen in pure nickel." Acta Materialia 60.19 (2012): 68146828

5. Liu, Qinglong, et al. "Hydrogen trapping in some advanced high strength steels." Corrosion Science 111 (2016): 770-785

6. Oriani, Richard A. "The diffusion and trapping of hydrogen in steel." Acta metallurgica 18.1 (1970): 147-157

7. McNabb, A., and P. K. Foster. "A New Analysis of Diffusion of Hydrogen in Iron and Ferritic Steels." Transactions of the Metallurgical Society of AIME 227.3 (1963): 618

8. Paul, Aloke, et al. Thermodynamics, diffusion and the Kirkendall effect in solids. Cham: Springer International Publishing, 2014

9. Larcht'e, F. C., and J. L. Cahn. "The effect of selfstress on diffusion in solids." Acta Metallurgica 30.10 (1982): 1835-1845

10. Birnbaum, Howard K., and Petros Sofronis. "Hydrogen-enhanced localized plasticity-a mechanism for hydrogen-related fracture."
Materials Science and Engineering: A 176.1-2 (1994): 191-202

11. Sofronis, Petros, Yueming Liang, and Nikolaos Aravas. "Hydrogen induced shear localization of the plastic flow in metals and alloys." European Journal of Mechanics-A/Solids 20.6 (2001): 857-872

12. Zhang, Zhenbo, et al. "On the role of precipitates in hydrogen trapping and hydrogen embrittlement of a nickel-based superalloy." Corrosion Science 146 (2019): 58-69

13. Frolova, Ksenia, et al. "Modelling of a hydrogen saturated layer within the micropolar approach." New Achievements in Continuum Mechanics and Thermodynamics. Springer, Cham, 2019. 117-128 